\title{
Rates of Subsequent Shoulder Surgery Within Three Years for Patients Undergoing SLAP Repair Versus Biceps Tenodesis
}

\author{
Ravand S. Khazai, M.D., Cody S. Lee, B.S., Haroutioun H. Boyajian, M.D., \\ Lewis L. Shi, M.D., and Aravind Athiviraham, M.D.
}

\begin{abstract}
Purpose: To investigate the demographics of patients for whom SLAP repair or biceps tenodesis was performed, as well as to compare rates of additional shoulder surgery for these 2 procedures within 3 years postoperatively. Methods: Using the MarketScan Commercial Database, we examined all patients with SLAP tear who underwent arthroscopic SLAP repair or open or arthroscopic biceps tenodesis within the encompassed time period (2003-2014). Rates of repeat shoulder surgery within 3 years were evaluated, as were comparative demographics. Results: In total, 25,142 patients initially underwent SLAP repair, of whom $11.5 \%$ had subsequent shoulder surgery within 3 years. A total of 840 patients initially underwent biceps tenodesis as treatment for a SLAP tear, of whom 13.0\% underwent additional shoulder surgery within 3 years. Rates of subsequent shoulder surgery between the 2 procedural groups did not statistically differ $(P=.19)$. Patients who underwent SLAP repair were younger than those who underwent tenodesis (mean age 38.3 vs 49.3 years, $P<.01$ ). For patients requiring additional surgery, the SLAP repair group had a greater representation of those $\geq 35$ years old, whereas the tenodesis group had a greater representation of those $<35$ years old $(P<.01)$. Male patients experienced an increase in rate of subsequent shoulder surgery when initially undergoing tenodesis versus SLAP repair (13.3\% vs $11.1 \%$, $P<.01)$. Conclusions: The rates of additional shoulder surgery for patients undergoing SLAP repair and biceps tenodesis were similar within 3 years of the index procedure. Patients who underwent SLAP repair were younger than those who underwent tenodesis. Of those requiring additional surgery, patients initially treated with SLAP repair were older $(\geq 35$ years) and those treated with tenodesis were younger $(<35$ years). Male patients experienced an increase in rate of subsequent shoulder surgery when initially treated with tenodesis versus SLAP repair. Level of Evidence: III, retrospective comparative study.
\end{abstract}

A lthough SLAP tears are relatively uncommon injuries overall, as diagnostic modalities become more sophisticated and sensitive, they are being

From the Department of Orthopaedic Surgery and Rehabilitation Medicine, University of Chicago Medicine, Chicago, Illinois (R.S.K., C.S.L., L.L.S., A.A.); and Department of General Surgery, Henry Ford Wyandotte Hospital, Wyandotte, Michigan (H.H.B.), U.S.A.

The authors report the following potential conflicts of interest or sources of funding: L.L.S. reports personal fees from DePuy Synthes, outside the submitted work. Full ICMJE author disclosure forms are available for this article online, as supplementary material.

Received August 31, 2019; accepted January 13, 2020.

Address correspondence to Aravind Athiviraham, M.D., Department of Orthopaedic Surgery and Rehabilitation Medicine, University of Chicago Medicine, 5841 S. Maryland Ave., MC 3079, Chicago, IL 60637. E-mail: cslee@uchicago.edu

(C) 2020 THE AUTHORS. Published by Elsevier Inc. on behalf of the Arthroscopy Association of North America. This is an open access article under the CC BY-NC-ND license (http://creativecommons.org/licenses/by-nc-nd/4.0/). 2666-061X/191062

https://doi.org/10.1016/j.asmr.2020.01.003 increasingly diagnosed. ${ }^{1,2}$ This increased rate of diagnosis has led to an increase in the number of patients treated surgically. ${ }^{1-5}$ However, there is no clear consensus on the optimal surgical management of SLAP tears. ${ }^{5,6}$ Debridement alone has been shown to have inconsistent outcomes, and so standard surgical treatment has typically involved repair of the SLAP lesion. ${ }^{7,8}$ Reported success rates, return to sport, and patient outcomes have been inconsistent following SLAP repair. ${ }^{2,5,6,8-10}$ Gorantla et al. ${ }^{11}$ published a systematic review that reported success rates of SLAP repair range from $40 \%$ to $94 \%$ and return to sport from $20 \%$ to $94 \% .^{11}$

Other studies have examined alternative surgical treatments, in particular biceps tenodesis, which has been described as effective both in the primary setting or as a salvage option for failed previous SLAP repair. ${ }^{2,6,9,12-19}$ In a cohort study of 25 patients, Boileau et al. ${ }^{9}$ reported greater improvement in satisfaction for tenodesis compared with repair $(93 \%$ to $40 \%$ ), as 
well as a greater rate of return to previous level of sporting activity ( $87 \%$ to $20 \%$ ). Denard et al. ${ }^{12}$ showed in another small series a greater rate of return to activity and satisfaction, as well as a shorter recovery time, for tenodesis when compared with repair in patients older than 35 years. A review by Huri et al. ${ }^{2}$ reiterates inconsistent outcomes with SLAP repair and hypothesizes that poor reliability and dissatisfaction with the procedure have contributed to the increasing incidence of tenodesis compared to direct repair. The reported number of biceps tenodeses performed as treatment for SLAP lesions has been increasing over the last 10 years, particularly for older patients, whereas the incidence of SLAP repairs has proportionally decreased over time. ${ }^{14-17}$

With variable reliability and concerns regarding need for additional surgery for treatment of SLAP tears, rates of subsequent shoulder surgery following these procedures are of particular interest. Mollon et al., ${ }^{20}$ in a recent case-control series, reported a $10.1 \%$ rate of subsequent shoulder surgery for patients undergoing isolated arthroscopic SLAP repair within 3 years, with many patients requiring more than one additional procedure. Rates of subsequent surgery following tenodesis have not been as well-investigated but are also of interest, especially given its acceptance and growing popularity as a surgical treatment.

The purpose of this study was to investigate the demographics of patients for whom SLAP repair or biceps tenodesis were performed, as well as to compare rates of additional shoulder surgery for these 2 procedures within 3 years postoperatively. We hypothesized that SLAP repairs would be associated with a greater rate of revision surgery within 3 years after initial procedure.

\section{Methods}

A retrospective review of the Truven Health MarketScan Commercial Database (IBM Watson Health, Ann Arbor, MI) was conducted to obtain the study's sample set. This database houses deidentified records for approximately 55 million patients in the United States younger than 65 years old with private or employer-provided coverage, spanning from 2003 to 2014. Records include, among other information, general demographics, payer data, International Classification of Diseases, Ninth Revision (ICD-9) diagnosis codes, billed costs for medications and office visits, and claims for Current Procedural Terminology (CPT) codes with associated dates. This study received no external funding and, given the deidentified nature of the data set, was exempted by the institutional review board.

Data were reviewed for patients with SLAP tears surgically treated between 2003 and 2014 by either arthroscopic SLAP repair (CPT code 29807) or biceps tenodesis (open or arthroscopic, CPT 23430 or 29828, respectively). Patients were excluded if they did not
Table 1. List of CPT Codes and Procedure Names Defined as Subsequent Shoulder Surgeries

\begin{tabular}{cl}
\hline CPT & \multicolumn{1}{c}{ Procedure } \\
\hline 29807 & Repair of SLAP lesion, arthroscopic \\
23430 & Biceps tenodesis, open \\
29828 & Biceps tenodesis, arthroscopic \\
29822 & Shoulder, limited debridement, arthroscopic \\
29823 & Shoulder, extensive debridement, arthroscopic \\
29825 & Shoulder, lysis of adhesions $+/-$ manipulation, arthroscopic \\
\hline CPT, & Current Procedural Terminology.
\end{tabular}

remain enrolled in the database for at least 3 consecutive years following their operation or if the CPT code of the procedure was not associated with an ICD-9 diagnosis code of SLAP tear (840.7). Patients who underwent a concomitant rotator cuff repair at the time of initial procedure (CPT 29827) also were excluded, to eliminate bias associated with SLAP repair or tenodesis being performed as part of a larger surgery.

Demographic data related to patient sex and age for each procedural group were then gathered, as were rates of subsequent shoulder surgery. Patients were determined to have had subsequent shoulder surgery if there was documentation of any of the CPT codes listed in Table 1 within the monitored 3-year postoperative period from their initial index procedure. The CPT codes included were limited to those that may have been related to the initial procedure. These additional surgeries were further analyzed by time from index procedure and stratified by patient sex and age (older or younger than 35 years, as described previously in the literature ${ }^{12,13}$ ).

A sample size calculation was conducted using conventional values of 0.80 for power and 0.05 for alpha. With literature reported rates of $10.1 \%$ of subsequent shoulder surgery for patients undergoing isolated arthroscopic SLAP repair within 3 years ${ }^{20}$ and approximately $15 \%$ for patients with primary proximal biceps tendon pathology for whom tenodesis is performed, ${ }^{21-23}$ a minimum sample size of 12,090 patients $(11,700$ with isolated SLAP repair and 390 with tenodesis) was required.

Demographic and clinical data were summarized using descriptive statistics. Patient sex, age $<$ or $\geq 35$ years, and subsequent surgery rates are presented as proportions and were statistically compared using $\chi^{2}$ analysis. Patient age and time to revision are presented as mean \pm standard deviation and were statistically compared using Student $t$ test. Significance was set at $P<.05$ for all statistical testing.

\section{Results}

From 2003 to 2014, a total of 25,142 patients underwent primary SLAP repair and maintained enrollment in the database for 3 years postoperatively. In the 
Table 2. Demographics of Patients Undergoing SLAP Repair and Biceps Tenodesis for SLAP Lesions Who Maintained 3-Year Database Follow-up and Were Included for Analysis

\begin{tabular}{lccc}
\hline & SLAP Repair & Tenodesis & $P$ Value \\
\hline Patients, $\mathrm{n}$ & 25,142 & 840 & \\
Male, $\mathrm{n}(\%)$ & $18,447(73.4 \%)$ & $617(73.5 \%)$ & .96 \\
Female, $\mathrm{n}(\%)$ & $6695(26.6 \%)$ & $223(26.5 \%)$ & .96 \\
Age, y, mean \pm SD & $38.3 \pm 14.0$ & $49.3 \pm 10.4$ & $<.01$ \\
$\mathrm{n}<35 \mathrm{y}, \mathrm{n}(\%)$ & $9170(36.5 \%)$ & $55(6.5 \%)$ & \\
$\mathrm{n} \geq 35 \mathrm{y}, \mathrm{n}(\%)$ & $15,963(63.5 \%)$ & $785(93.5 \%)$ & $<.01$ \\
\hline
\end{tabular}

SD, standard deviation.

same time period, a total of 15,173 patients underwent biceps tenodesis and maintained adequate enrollment. However, only 840 of these patients carried an associated diagnosis code of SLAP lesion linked to their surgery and were included in the study (Table 2). There was no difference in the breakdown of male and female patients between procedural groups $(P=.96)$. Patients who underwent SLAP repair were younger than those who underwent tenodesis (mean age 38.3 vs 49.3 years, $P<.01$ ). This was further highlighted by the greater representation of patients younger than the age of 35 years in the group who underwent SLAP repair versus tenodesis $(36.5 \%$ vs $6.5 \%, P<.01)$.

Overall rates of subsequent shoulder surgery did not statistically differ between patients who initially had SLAP repair versus tenodesis 3 years postoperatively (11.5\% vs $13.0 \%$, respectively, $P=.19$ ) (Table 3 ). Furthermore, cumulative rates of additional shoulder surgery did not differ between the 2 procedural groups at any time point measured in 6-month time periods post-initial procedure (Fig 1). In addition, the proportion of total subsequent shoulder surgeries performed for both treatment groups did not statistically differ for any 6-month time period (Fig 2). Patients requiring additional surgery experienced a shorter time to the operating room if initially treated by tenodesis versus SLAP repair (312.0 vs 410.1 days, $P<.01)$.

When stratified by sex, the rate of subsequent shoulder surgery was statistically greater for male patients who initially underwent tenodesis versus those who underwent SLAP repair $(13.3 \%$ vs $11.1 \%$,
$P<.01)$. Conversely, there was no significant difference in the rate of additional surgery for female patients between the 2 procedures $(P=.83)$. When looking at only patients who underwent additional surgery, the ratio of male to female patients did not differ between SLAP repair and tenodesis groups $(P=.32)$.

Patients aged $<35$ years did not experience a significant difference in rate of subsequent shoulder surgery between treatment groups $(P=.15)$. The same was true for patients aged $\geq 35$ years $(P=.59)$. However, for those patients who underwent additional surgery, those treated with tenodesis were younger than those treated with SLAP repair, as the representation of patients $<35$ years old was statistically significantly greater in the tenodesis group versus the SLAP group $(P<.01)$.

\section{Discussion}

Contrary to our hypothesis, the current study found no significant difference in rate of subsequent shoulder surgery between patients initially treated with SLAP repair and biceps tenodesis within 3 years of initial procedure. In addition, there was no observed difference in the rate of change of subsequent surgery over the 3-year period between the 2 procedural groups. Our hypothesis was generated based on the previous findings in the literature regarding the inconsistent outcomes of SLAP repair and increased rate of return to activity and patient satisfaction for biceps tenodesis. $2,9,12,24$ However, in an effort to challenge previous study findings, a randomized, sham-controlled, clinical trial by Schroder et al. questioned the efficacy of either

Table 3. Rates of Subsequent Shoulder Surgery Within 3 Years for Patients Initially Undergoing SLAP Repair Versus Biceps Tenodesis for SLAP Lesion

\begin{tabular}{|c|c|c|c|}
\hline & SLAP Repair & Tenodesis & $P$ Value \\
\hline Overall subsequent surgery rate & $11.5 \%$ & $13.0 \%$ & .19 \\
\hline Time to subsequent surgery, $\mathrm{d}$, mean $\pm \mathrm{SD}$ & $410.1 \pm 329.8$ & $312.0 \pm 309.7$ & $<.01$ \\
\hline \multicolumn{4}{|l|}{ Rate by sex } \\
\hline Female & $12.6 \%$ & $12.1 \%$ & .83 \\
\hline \multicolumn{4}{|l|}{ Rate by age } \\
\hline$<35 \mathrm{y}$ & $10.4 \%$ & $16.4 \%$ & .15 \\
\hline
\end{tabular}

SD, standard deviation. 


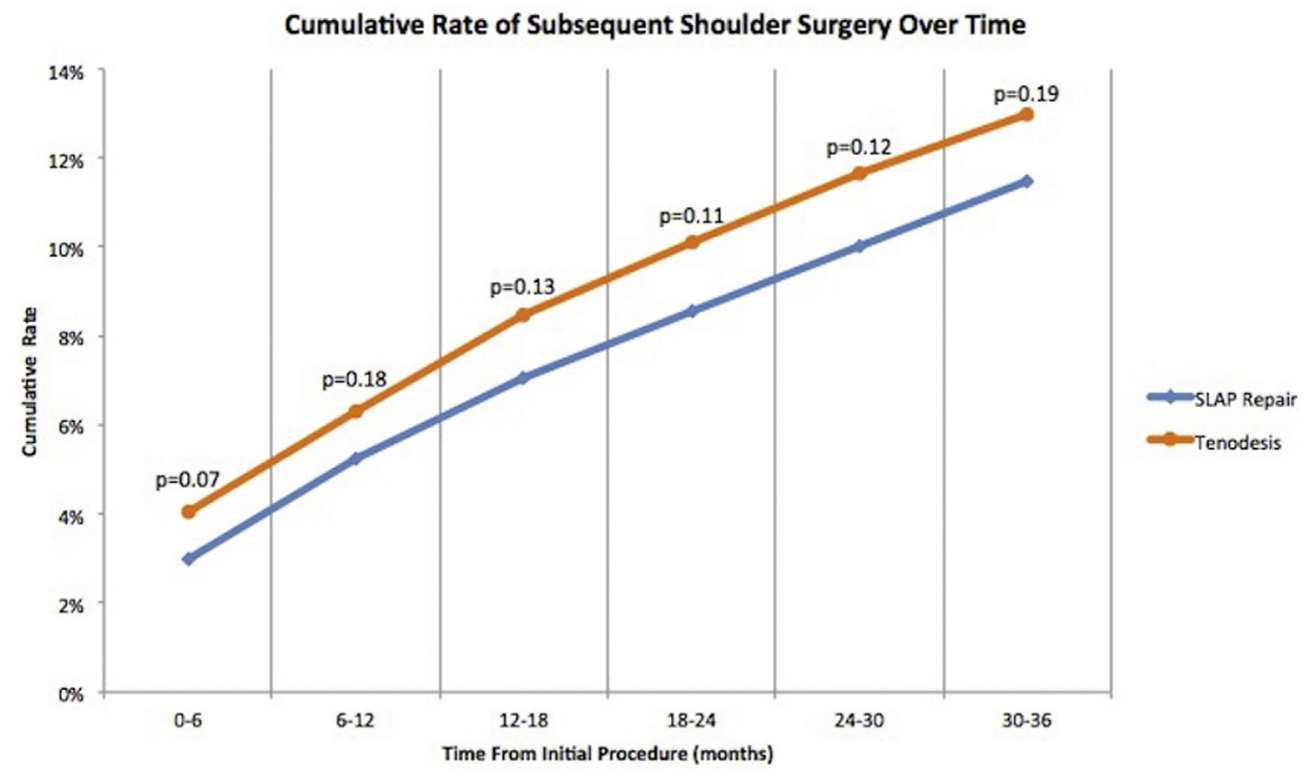

Fig 1. Cumulative rates of subsequent shoulder surgery for each procedural group were statistically compared at 6-month intervals from initial index procedure. Rates did not statistically differ at any measured time point.

mode of surgical management, be it repair or tenodesis, for SLAP lesions to affect return to activity or patient satisfaction. ${ }^{25,26}$ After evaluating objective and subjective patient outcomes at 6 and 24 months for those undergoing SLAP repair, tenodesis, or shamarthroscopic surgery, Schroder et al. ${ }^{25}$ concluded that neither labral repair nor biceps tenodesis had any significant clinical benefit over sham surgery for patients with SLAP lesions in the population studied. Although this study seems to suggest that nonoperative treatment has a good probability of success, outcomes of revision surgery or need for additional procedures were not studied.

In 2 recently published systematic reviews and metaanalyses, by Hurley et al. ${ }^{27}$ and Li et al., ${ }^{24}$ patient satisfaction and return to sport were analyzed in conjunction with rates of additional surgery. Patient satisfaction and return to sport were significantly better for patients undergoing tenodesis versus SLAP repair, but no difference was observed in rates of additional surgery between groups. The results of our current study support the study findings of Hurley et al. ${ }^{27}$ and Li et al. ${ }^{24}$ and suggest that although tenodesis may prove superior to SLAP repair for certain patient outcome measures, the rates of subsequent shoulder surgery between groups do not significantly differ in aggregate. Future sham-controlled, clinical studies investigating SLAP repair and tenodesis with subjective and objective outcomes, such as patient satisfaction and return to activity, should also incorporate the

Fig 2. The proportion of total subsequent shoulder surgeries completed for each procedural group was statistically compared at 6-month intervals from initial index procedure. No significant differences were observed between the 2 groups.

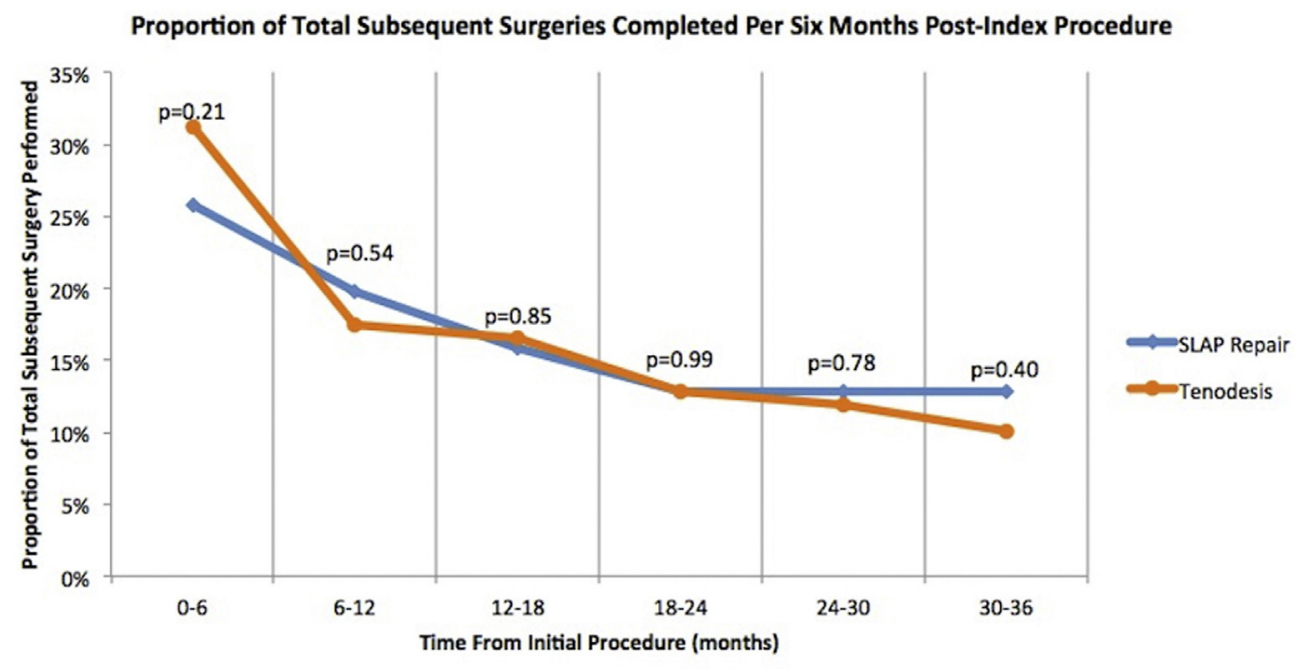


comparison of need for future surgical procedures in sham-controlled groups to that of the procedural groups.

Our overall rate of subsequent shoulder surgery for patients who underwent initial SLAP repair was $11.5 \%$. This is similar to previously cited rates in the literature. Mollon et al. ${ }^{20}$ performed a recent review of 2524 patients who had undergone arthroscopic SLAP repair in New York State and found a $10.1 \%$ rate of subsequent ipsilateral shoulder surgery within 3 years. This review was subject to possible selection bias as its sample was restricted to a single state, and practice trends may vary regionally. The advantage of our study was its use of a large-scale database for its sample, allowing for a large, as well as geographically diverse, data set.

Of patients who underwent biceps tenodesis for treatment of SLAP tear, our study found a $13.0 \%$ rate of additional shoulder surgery within 3 years. Although several studies have examined patient outcomes following SLAP-related biceps tenodesis, rate of subsequent surgery for the procedure has been less well described. In patients with primary proximal biceps tendon pathology for whom tenodesis is performed, rates of additional ipsilateral shoulder surgery have been reported at approximately $15 \% .{ }^{21-23}$ Although this historical rate is similar to the figure in our study, these previous studies have not specifically examined biceps tenodesis performed for the management of SLAP tears. We attempted to isolate this particular population in our study by only including those patients with an associated diagnosis code of SLAP tear linked to their tenodesis procedure.

Of those patients that required additional surgery, the SLAP repair group had a greater representation of those $\geq 35$ years old, whereas the tenodesis group had a greater representation of those $<35$ years old. In addition, those initially treated with SLAP repair were significantly younger than those treated with tenodesis. The study is consistent with a retrospective analysis by Ek et al., ${ }^{13}$ which concluded that tenodesis was performed in older patients ( $>35$ years) and those who showed degenerative or frayed labrums, whereas repair was performed in younger and more active patients with healthy-appearing labral tissue, despite finding no differences in patient satisfaction or return to activity between procedural groups. The age-related differences among treatment groups determined in our study also reflect and support current practice trends. Erickson et al. ${ }^{15}$ report in a systematic review a significantly greater rate of failure of SLAP repairs in patients older than 40 years. The literature accepts age as a risk factor for the development of postoperative stiffness and surgical failure ${ }^{11,16,17}$ and suggests tenodesis for the management of SLAP lesions for older patients. This is true in particular with younger sports surgeons. Weber et al. $^{5}$ examined the American Board of Orthopedic
Surgery database and found that for part II applicants, reported rates of SLAP repair were 3 times greater than seen in previous literature, with rates increasing over time. Patterson et al. ${ }^{17}$ queried the same database, and noted an increased proportion of these lesions treated with biceps tenodesis, in particular with older patients. This pattern appears to be consistent with other studies. ${ }^{12,14}$ The clinical relevance of this difference in rates is a topic of further investigation.

Although the male-to-female breakdown was statistically similar between the 2 procedural groups, there was an observed statistically significant increase in rate of subsequent shoulder surgery for male patients initially treated with tenodesis versus SLAP repair. To our knowledge, a significant difference in this rate between treatment types based solely on patient sex has not been reported. Because there was no difference in male-to-female ratio between treatment types for those patients requiring an additional surgery, it is possible that the observed difference in rate for male patients could be contributed to a confounding factor. It is possible that the male patients in the tenodesis group were younger in comparison with those in the SLAP repair group, which could account for the observed difference, assuming our difference in results for each procedural group based on age is significant.

Analyzing large, population-based databases can be challenging; however, the use of the MarketScan Commercial Database in our study has several strengths. It contains 12 consecutive years of data from a significant nationwide sample, accumulating and capturing data of nearly 55 million Americans. This allows not only analysis of a large sample but also avoids institution and protocol-related biases inherent in single-center series.

However, there are limitations inherent to any database study, and some characteristic to the MarketScan Commercial Database. All diagnoses used for this study were derived from documented CPT and ICD-9 codes. Our study is therefore subject to errant coding and would fail to capture patients that had pathology coded differently than the ICD-9 code for SLAP tear. This is particularly relevant for the group of patients who initially underwent tenodesis because a significant number of them may have had SLAP lesions, but were documented using more generic diagnosis codes, eliminating them from inclusion in our study. This potential mode of exclusion is one possible explanation for the disparity in patient numbers between procedural groups. In addition, if tenodesis were more commonly performed in those undergoing concomitant rotator cuff repair, this would also contribute to the large difference in patient numbers between groups, as subjects were excluded if their initial SLAP-related procedure was performed along with rotator cuff repair. Although this was done to eliminate bias 
associated with SLAP repair or tenodesis being performed as part of a larger surgery and being inappropriately compared to isolated repair or tenodesis, the present study did not analyze differences in SLAP tear type, concomitant pathology, or concomitant procedures between groups, which could affect the rates of additional shoulder surgery. The MarketScan Commercial Database is also limited to privately insured patients. Those with Medicare without supplemental private insurance are not included in this database, which may allow for selection bias and limits generalizability of the data. In addition, the database used for this study did not include potentially pertinent patient health risk data that could affect rates of additional surgery, such as biometrics and health behaviors (i.e., body mass index, patient activity level, smoking status, blood glucose levels, etc.). Future studies can take advantage of the MarketScan Health Risk Assessment Database to analyze these factors' influence on rates of additional shoulder surgery.

\section{Conclusions}

The rates of additional shoulder surgery for patients undergoing SLAP repair and biceps tenodesis were similar within 3 years of the index procedure. Patients who underwent SLAP repair were younger than those who underwent tenodesis. Of those requiring additional surgery, patients initially treated with SLAP repair were older ( $\geq 35$ years) and those treated with tenodesis were younger ( $<35$ years). Male patients experienced an increase in rate of subsequent shoulder surgery when initially treated with tenodesis versus SLAP repair.

\section{References}

1. Blaine TA, Edwards S, Lee J, et al. Improved outcomes with non-operative treatment of superior labral tears. Arthroscopy 2007;23:e27.

2. Huri G, Hyun YS, Garbis NG, et al. Treatment of superior labrum anterior posterior lesions: A literature review. Acta Orthop Traumatol Turc 2014;48:290-297.

3. Katz LM, Hsu S, Miller SL, et al. Poor outcomes after SLAP repair: Descriptive analysis and prognosis. Arthroscopy 2009;25:849-855.

4. Zhang AL, Kreulen C, Ngo S, et al. Demographic trends in arthroscopic SLAP repair in the United States. Am J Sports Med 2012;40:1144-1147.

5. Weber SC, Martin DF, Seiler JG, et al. Superior labrum anterior and posterior lesions of the shoulder: incidence rates, complications, and outcomes as reported by American Board of Orthopedic Surgery Part II Candidates. Am J Sports Med 2012;40:1538-1543.

6. Weber SC. Surgical management of the failed SLAP repair. Sports Med Arthrosc 2010;18:162-166.

7. Cordasco FA, Steinmann S, Flatow EL, et al. Arthroscopic treatment of glenoid labral tears. Am J Sports Med 1993;21: 425-430.
8. Kim SH, Ha KI, Kim SH, et al. Results of arthroscopic treatment of superior labral lesions. J Bone Joint Surg Am 2002;84-A:981-985.

9. Boileau P, Parratte S, Chuinard C, et al. Arthroscopic treatment of isolated type II SLAP lesions: Biceps tenodesis as an alternative to reinsertion. Am J Sports Med 2009;37:929-936.

10. Jang SH, Seo JG, Jang HS, et al. Predictive factors associated with failure of nonoperative treatment of superior labrum anterior-posterior tears. J Shoulder Elbow Surg 2016;25:428-434.

11. Gorantla K, Gill C, Wright RW. The outcome of type II SLAP repair: A systematic review. Arthroscopy 2010;26:537-545.

12. Denard PJ, Lädermann A, Parsley B, et al. Arthroscopic biceps tenodesis compared with repair of isolated type II SLAP lesions in patients older than 35 years. Orthopedics 2014;37:e292-e297.

13. Ek ET, Shi LL, Tompson JD, et al. Surgical treatment of isolated type II superior labrum anterior-posterior (SLAP) lesions: Repair versus biceps tenodesis. J Shoulder Elbow Surg 2014;23:1059-1065.

14. Erickson BJ, Jain A, Abrams GD, et al. SLAP lesions: Trends in treatment. Arthroscopy 2016;32:976-981.

15. Erickson BJ, Lavery K, Monica J, et al. Surgical treatment of symptomatic superior labrum anterior-posterior tears in patients older than 40 years: A systematic review. Am J Sports Med 2015;43:1274-1282.

16. Gottschalk MB, Karas SG, Ghattas TN, et al. Subpectoral biceps tenodesis for the treatment of type II and IV superior labral anterior and posterior lesions. Am J Sports Med 2014;42:2128-2135.

17. Patterson BM, Creighton RA, Sprang JT, et al. Surgical trends in the treatment of superior labrum anterior and posterior lesions of the shoulder: Analysis of data from the American Board of Orthopaedic Surgery Certification Examination Database. Am J Sports Med 2014;42: 1904-1910.

18. Sanders B, Lavery KP, Pennington S, Warner JJ. Clinical success of biceps tenodesis with and without release of the transverse humeral ligament. J Shoulder Elbow Surg 2012;21:66-71.

19. Werner BC, Pehlivan HC, Brockmeier SF, et al. Biceps tenodesis is a viable option for salvage of failed SLAP repair. J Shoulder Elbow Surg 2014;23:e179-el84.

20. Mollon B, Mahure SA, Ensor KL, et al. Subsequent shoulder surgery after isolated arthroscopic SLAP repair. Arthroscopy 2016;32:1954-1962.

21. Becker DA, Cofield RH. Tenodesis of the long head of the biceps brachii for chronic bicipital tendinitis. Long-term results. J Bone Joint Surg Am 1989;71:376-381.

22. Gill TJ, McIrvin E, Mair SD, Hawkins RJ. Results of biceps tenotomy for treatment of pathology of the long head of the biceps brachii. J Shoulder Elbow Surg 2001;10:247-249.

23. Kim SJ, Lee IS, Kim SH, et al. Arthroscopic repair of concomitant type II SLAP lesions in large to massive rotator cuff tears: Comparison with biceps tenotomy. Am J Sports Med 2012;40:2786-2793.

24. Li M, Shaikh AB, Sun J, et al. Effectiveness of biceps tenodesis versus SLAP repair for surgical treatment of isolated SLAP lesions: A systemic review and meta-analysis. J Orthop Translat 2019;16:23-32. 
25. Schroder CP, Skare O, Reikeras O, et al. Sham surgery versus labral repair or biceps tenodesis for type II SLAP lesions of the shoulder: A three-armed ransomised clinical trial. Br J Sports Med 2017;51:1759-1766.

26. Skare O, Schroder CP, Reikeras O, et al. Efficacy of labral repair, biceps tenodesis, and diagnostic arthroscopy for
SLAP lesions of the shoulder: A randomised controlled trial. BMC Musculoskelet Disord 2010;11:228.

27. Hurley ET, Fat DL, Duigenan CM, et al. Biceps tenodesis versus labral repair for superior labrum anterior-toposterior tears: A systematic review and meta-analysis. J Shoulder Elbow Surg 2018;27:1913-1919. 